\title{
Two-Dimensional Rotating Stall Analysis in a Wide Vaneless Diffuser
}

\author{
S. Ljevar, ${ }^{1,2}$ H. C. de Lange, ${ }^{1}$ and A. A. van Steenhoven ${ }^{1}$ \\ ${ }^{1}$ Section of Energy Technology, Division Thermo Fluids Engineering, Department of Mechanical Engineering, \\ Eindhoven University of Technology, Den Dolech 2, 5600 MB Eindhoven, The Netherlands \\ ${ }^{2}$ Department of Flow and Structural Dynamics, TNO Science and Industry, P.O. Box 155, 2600 AD Delft, The Netherlands
}

Received 26 August 2005; Accepted 7 September 2005

\begin{abstract}
We report a numerical study on the vaneless diffuser core flow instability in centrifugal compressors. The analysis is performed for the purpose of better understanding of the rotating stall flow mechanism in radial vaneless diffusers. Since the analysis is restricted to the two-dimensional core flow, the effect of the wall boundary layers is neglected. A commercial code with the standard incompressible viscous flow solver is applied to model the vaneless diffuser core flow in the plane parallel to the diffuser walls. At the diffuser inlet, rotating jet-wake velocity pattern is prescribed and at the diffuser outlet constant static pressure is assumed. Under these circumstances, two-dimensional rotating flow instability similar to rotating stall is found to exist. Performed parameter analysis reveals that this instability is strongly influenced by the diffuser geometry and the inlet and outlet flow conditions.
\end{abstract}

Copyright (c) 2006 S. Ljevar et al. This is an open access article distributed under the Creative Commons Attribution License, which permits unrestricted use, distribution, and reproduction in any medium, provided the original work is properly cited.

\section{INTRODUCTION}

The performance of compressors at low mass-flows is characterized by the occurrence of unsteady flow phenomena surge and rotating stall. These instabilities can cause noise nuisance and critical operating conditions with strong dynamical loading on the blades. Therefore, they cannot be tolerated during compressor operation. To avoid these phenomena, compressors are operated at higher mass-flow rates than those prevailing at the critical operating conditions to keep a safety margin to the stability limit. Limiting of the compressor operating range results in the loss of high-pressure ratios, which makes this issue an interesting subject to research.

Main effort of this research is concentrated on the development of surge and stall control systems in centrifugal compressors. After Willems [1] has designed and implemented a model for active surge control on a small-scale centrifugal compressor, rotating stall turned out to occur when surge was postponed. In order to increase the region of operation of centrifugal compressors, the understanding of rotating stall flow dynamics is required.

The most common type of rotating stall in centrifugal compressors is the diffuser rotating stall. This paper deals with the study of rotating stall mechanism within the vaneless radial diffusers. In the literature, different analytical and experimental approaches have been used to investigate the rotating stall phenomenon, and several theories that explain the vaneless diffuser rotating stall mechanism have been developed. The literature survey on the vaneless diffuser rotating stall indicates that two or maybe more different flow mechanisms can be responsible for the occurrence of rotating stall in vaneless radial diffusers. Abdelhamid and Bertrand [2], Dou [3], and Shin et al. [4] have found that vaneless diffuser performance is different for narrow and wide diffusers and they clearly suggest that different flow mechanisms might exist and can lead to the occurrence of rotating stall. In general, one mechanism is associated with the twodimensional core flow instability occurring in wide vaneless diffusers when the critical flow angle is reached and the other mechanism is associated with the three-dimensional wall boundary layer instability occurring in the narrow diffusers.

Three-dimensional approach where the wall boundary layer theory is used to study the rotating stall in vaneless diffusers was applied by Jansen [5], Senoo and Kinoshita [6], Frigne and Van den Braembussche [7], and Dou and Mizuki [8], among others. They generally hold the effect of the threedimensional boundary layers near the walls responsible for the occurrence of rotating stall in vaneless diffuser. On the other hand, Abdelhamid [9] and Tsujimoto et al. [10] have used a two-dimensional approach where the effect of the wall boundary layers is not taken into account. They both used a two-dimensional inviscid and incompressible flow analysis 
to study the vaneless diffuser rotating stall. These studies suggest the existence of a two-dimensional core flow instability at the onset of rotating stall in vaneless diffusers.

Additionally, measurements of rotating stall in radial vaneless diffusers are performed by Abdelhamid and Bertrand [2], Abdelhamid [11], Frigne and Van den Braembussche [7], Dou [3], Shin et al. [4], Ferrara et al. [12, 13], and Cellai et al. [14, 15]. Experimental work shows significant influence of the diffuser geometry on the vaneless diffuser performance and on the structure of the rotating stall pattern.

Previous studies indicate that rotating stall mechanism is still obscure and that further research is necessary to reveal its flow dynamics. Since some literature suggests that the core flow instability can be one of the mechanisms causing the rotating stall in vaneless diffusers, rotating stall inception in vaneless diffuser is investigated from the point of view that it can be a two-dimensional flow instability. Therefore, the research is restricted only to the wide vaneless radial diffusers with parallel diffuser walls where it can be assumed that mainly two-dimensional core flow in between the diffuser plates is present.

In this paper, an instability analysis of the two-dimensional vaneless diffuser core flow is performed using CFD. Therefore, a two-dimensional incompressible flow model of the vaneless diffuser core flow is developed where the influence of the wall boundary layers is not taken into account. Therefore, this model applies only to the wide vaneless radial diffusers with parallel diffuser walls. Since wide vaneless diffusers that compare well with this numerical model in [16] all satisfy $h / r_{2}>0.1$, all vaneless diffusers that satisfy this condition will be considered as wide vaneless diffusers.

This analysis studies the influence of geometry parameters and diffuser inlet flow conditions on the two-dimensional vaneless diffuser flow stability. To study the effect of the diffuser inlet flow conditions, the impeller tip speed, mass-flow rate, and shape and intensity of the jet-wake pattern at the diffuser inlet are varied. And to investigate the influence of the diffuser geometry, the diffuser radius ratio and the number of impeller blades are varied. The effect of these parameters on the core flow stability and the structure and behavior of the two-dimensional rotating instability is investigated.

This paper is divided into a number of sections. Section 2 describes the applied scaling procedure where the scaling of the existing air compressor into the water model of the wide vaneless diffuser is performed. Section 3 describes the texture of the numerical model and the main modeling aspects and Section 4 describes the obtained two-dimensional rotating instability and the accompanying stability criteria. In Section 5, the performed parameter analysis is discussed where the influence of different geometrical and flow parameters on the two-dimensional rotating instability is studied. Finally, in Section 6 the subject is closed with a summarizing and concluding discussion.

\section{SCALING}

This paper does not discuss any experimental research, but to understand the texture of the numerical model a few words are spent on the relation between the experimental and numerical model. To experimentally study the overall image of rotating stall, water model of the centrifugal compressor stage is built. The hydrodynamic analogy shows that a water model of the same geometry must operate at much lower fluid velocities and shaft speeds than the air compressor configuration. Deceleration of the process allows plain visualization of the unsteady flow phenomena using the particle image velocimetry. Therefore, scaling of the existing air compressor stage into a water model of the centrifugal compressor stage is performed and applied. The operating condition of an existing research air compressor near the stability limit is scaled to obtain a water model that operates near stalling conditions. Since the numerical model is destined to support the experimental study of rotating stall, it is based on the scaled diffuser geometry and fluid operating flow conditions.

To scale the working medium pressure rise across the air compressor, $p_{03} / p_{01}$, and the modeled water stage, $p_{3} / p_{1}$, is set equal. While scaling the working medium, it is assumed that the diffuser dimensions remain unchanged. According to Cohen et al. [17] the pressure rise across the air compressor can be expressed as

$$
\begin{aligned}
\frac{p_{03}}{p_{01}} & =\left[1+\frac{\eta_{c} \cdot\left(T_{03}-T_{01}\right)}{T_{01}}\right]^{\gamma /(\gamma-1)} \\
& =\left[1+\frac{\eta_{c} \cdot \psi \cdot \sigma \cdot v_{\text {tip }}^{2}}{c_{p} \cdot T_{01}}\right]^{\gamma /(\gamma-1)} .
\end{aligned}
$$

When the working medium is water, the pressure rise across the compressor is defined by the energy equation

$$
\frac{p_{3}}{p_{1}}=1+\frac{\rho \cdot \psi \cdot \sigma \cdot v_{\text {tip }}^{2}}{p_{1}}-\frac{\rho}{2 \cdot p_{1}} \cdot\left(V_{3}^{2}-V_{1}^{2}\right) .
$$

Near stall operating conditions of the modeled water compressor stage are obtained by substitution of the air compressor operating conditions at stall onset.

Subsequently, the dimensions of the modeled vaneless diffuser are scaled to the desired values. Therefore, the pressure gradient across the diffuser is kept constant, which is defined as follows:

$$
\frac{p_{3}-p_{2}}{r_{2}}=-\frac{1}{2} \cdot \frac{\rho}{r_{2}} \cdot\left(V_{3}^{2}-V_{2}^{2}\right) .
$$

Keeping the pressure gradient constant and applying the diffuser radius ratio $r_{3} / r_{2}=1.52$ gives the relation between the absolute velocity at the diffuser inlet and the diffuser inlet radius, $V_{2}^{2} / r_{2}=189.2$, which must apply to the new diffuser geometry. Furthermore, constant mean flow angle must apply and therefore the similarity of the velocity triangles is applied at the flow condition near the stability limit. The velocity triangle relations are used to determine the water model operating conditions.

To obtain exact similarity between the air compressor flow and the fluid flow within the modeled vaneless diffuser, Reynolds and Mach numbers must remain unchanged. Since the pressure gradient is kept constant during the scaling, it is not possible to also keep the Reynolds numbers exactly 


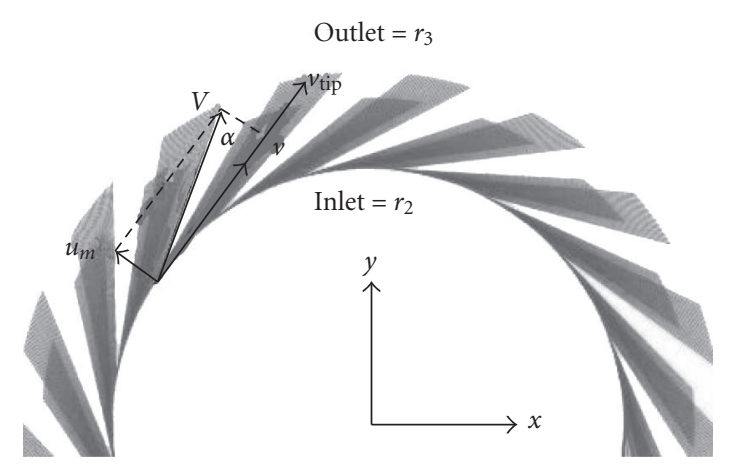

FiguRE 1: Diffuser model geometry and the boundary conditions.

the same in the water and air configuration. One can satisfy either the Reynolds number condition or the pressure gradient condition but not both conditions at the same time. Flow field similarity check shows that the Reynolds number, $\operatorname{Re}=r_{2} \cdot V / \nu$, in both configurations equals the order of $10^{5}$ or higher. The fluid flow as well as the air compressor flow is of a highly turbulent character. It is not possible to satisfy the Mach number similarity when water is used as working medium. In the air configuration $\mathrm{Ma}<1$, which means that the flow is subsonic. When the flow is subsonic, density changes over the compressor stage are small and the gas dynamic phenomena like shock waves do not have to be considered.

\section{NUMERICAL MODEL}

To study the core flow instability in a vaneless radial diffuser, two-dimensional flow in the plane parallel to diffuser walls is modeled. Consider a radial vaneless diffuser as shown in Figure 1 with finite outer to inner radius ratio $r_{3} / r_{2}$. Since the core flow of the wide vaneless diffuser is assumed to be twodimensional, no diffuser width is modeled and the influence of the wall boundary layers is not taken into account.

It is assumed that the outlet of the vaneless radial diffuser is connected to the space with constant static pressure. At the diffuser inlet, a clockwise rotating jet-wake velocity pattern is specified as shown in Figure 1. Prescribed tangential velocity component is constant around the circumference and is related to the impeller speed as follows:

$$
v=\frac{v_{\text {tip }}}{\sigma},
$$

where $v$ is the tangential velocity component, $v_{\text {tip }}$ impeller tip speed, and $\sigma$ slip factor. Radial velocity component at the diffuser inlet is described by the periodic hyperbolic tangent function,

$$
u=u_{m}+A \cdot \frac{\tanh (D \cdot Y)}{\tanh (D)}
$$

where $u$ is the radial velocity component, $u_{m}$ mean radial velocity, $A$ amplitude, and $D$ constant indicating the steepness of the jet-wake function. The circumferential position $\theta$ and impeller angular velocity $\omega_{i}$ are defined within the parameter Yas follows:

$$
Y=\sin \left(N \cdot \theta+\omega_{i} \cdot t\right)
$$

where $t$ is the time and $N$ is the number of jet-wakes around the circumference corresponding to the number of impeller blades.

The reference geometry and the operating conditions of the diffuser model as obtained by scaling the existing air compressor configuration at near stall operating conditions are $r_{2}=0.03225 \mathrm{~m} ; r_{3}=0.04908 \mathrm{~m} ; v_{\text {tip }}=2.6980 \mathrm{~m} / \mathrm{s}$, and $u_{m}=0.2905 \mathrm{~m} / \mathrm{s}$. The modeled number of impeller blades equals 17 . In the reference situation, jet-to-wake circumferential extent ratio equals one and the jet-to-wake radial intensity ratio equals 5.5 approximately.

For the numerical analysis a commercial software package FLUENT is used. The governing integral equations for the conservation of mass and momentum are solved using the finite-volume approach. For discretization of the timedependent terms, the second-order implicit time integration is used and for convection terms, the QUICK scheme as proposed by Leonard [18] is used. The QUICK scheme is thirdorder accurate for the spatial discretization of the convection terms and second-order accurate for the diffusion terms.

Although the studied flow is turbulent, an incompressible viscous flow solver is applied, which means that no eddy viscosity but only molecular viscosity is modeled. Turbulence models are not used in order to avoid the excessive numerical dissipation. It is assumed that the two-dimensional core flow instabilities have the length scale of the prescribed jetwake pattern at the diffuser inlet. Since turbulence models capture the diffusion-like character of turbulent mixing associated with many small eddy structures, they damp out the solutions of large eddy structures, like this one.

To mesh this geometry, a simple two-dimensional quadrilateral grid consisting of 750 by 62 elements is applied. The convergence criterion of $10^{-3}$ is applied to the continuity, $x$ velocity, and $y$-velocity residual. Under these conditions radial vaneless diffuser attributed core flow instability is studied.

\section{2D ROTATING INSTABILITY}

Numerical model of the vaneless diffuser core flow shows that vaneless diffusers have a two-dimensional rotating instability that is similar to rotating stall. Figure 2 shows two solutions corresponding to the reference operating flow conditions and geometry where solution in Figure 2(a) represents the steady stable operating flow condition and solution in Figure 2(b) shows the two-dimensional rotating instability. Solutions in Figure 2 are represented by the contours of velocity magnitude where the dark area inside the rotating cells represents high velocity and the dark areas near the diffuser outlet represent low-velocity magnitude. 


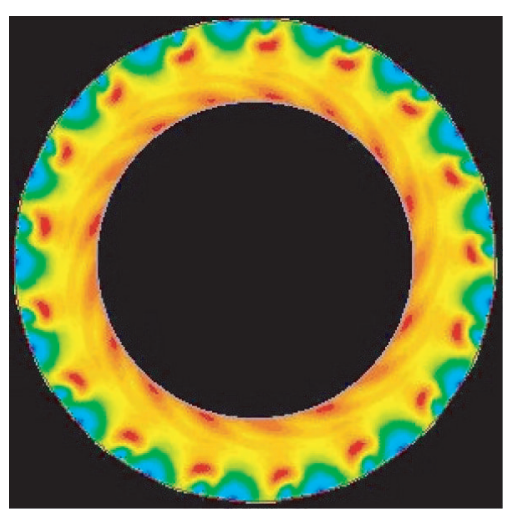

(a)

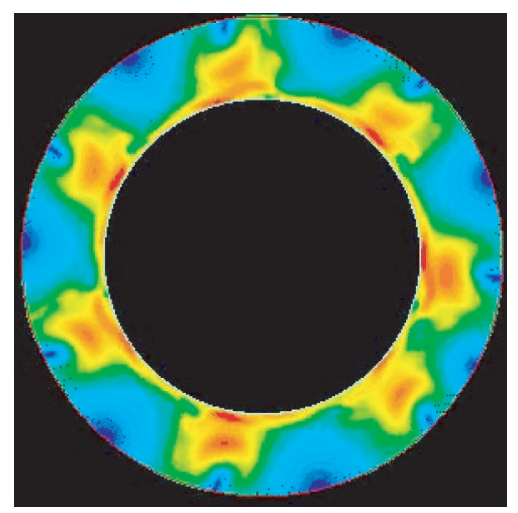

(b)

FIgURE 2: (a) Stable operating condition and (b) two-dimensional rotating instability.

\subsection{Similarity with rotating stall}

The obtained two-dimensional rotating instability is similar to rotating stall. It develops within four to eight impeller revolutions and consists of a number of rotating cells that are propagating with approximately $40 \%$ of the impeller speed. Besides the similarity with rotating stall based on the instability characteristics, numerical results also agree well with the measurements found in the literature for the same type of diffusers, as shown in [16]. Because of high similarity with rotating stall and good agreement with the measurements found in the literature, it is presumed that this instability might contribute to the vaneless diffuser rotating stall.

\subsection{Stability limit}

Within the two-dimensional diffuser core flow analysis, tangential velocity component $v$ and mean radial velocity component $u_{m}$ are varied to study their influence on the solution. By alternately varying the tangential and mean radial velocity component, a matrix of simulations is performed showing that the two-dimensional rotating instability occurs when $v / u_{m}$ ratio at the diffuser inlet exceeds a certain value. In Table 1 the stability limit is clearly visible. Since the $v / u$ ratio can be interpreted as the flow angle $\alpha$, the stability limit of the two-dimensional vaneless diffuser core flow can be expressed in terms of the critical flow angle at diffuser inlet, $\alpha_{\mathrm{cr}}$. The flow angle $\alpha$, as defined in Figure 1, is the angle between the absolute velocity and tangential velocity component. Therefore, the mean flow angle can be expressed as $\alpha_{m}=\tan ^{-1}\left(u_{m} / v\right)$. The core flow stability criterion suggests that the two-dimensional rotating instability will occur as soon as the mean flow angle at the diffuser inlet becomes smaller than the critical flow angle: $\alpha_{m}<\alpha_{\mathrm{cr}}$. The core flow is considered unstable as soon as the periodicity of the steady stable operating flow condition is disturbed. For the wide vaneless diffuser that satisfies the reference geometry and operating conditions and with the current assumptions at the diffuser inlet, outlet and walls it is found that $\alpha_{\mathrm{cr}}=6.8^{\circ}$. For different diffuser geometries and configurations the critical flow angle takes different values.

According to Table 1 mean flow angle decreases as the impeller speed increases or as the mean radial velocity component decreases. This model shows that the two-dimensional rotating instability occurs at small values of the mean flow angle. Compared to the existing air compressors this makes sense because in the real compressor configurations the mean flow angle also decreases towards the stalling conditions. The impeller speed usually remains unchanged while the mass-flow is being decreased which corresponds to the decrease of the radial velocity and thus the mean flow angle.

\subsection{Flow field analysis}

To understand the flow mechanisms behind this rotating instability, the flow field of the two-dimensional core flow is analyzed for the steady stable operating flow condition as well as for the two-dimensional rotating instability solution. To better distinguish the flow structures within the velocity field, relative velocity field is analyzed. Relative velocity field is obtained by subtracting the mean velocity, averaged along the circumference, from the absolute velocity field.

Relative velocity field of the steady stable operating flow condition is shown in Figure 3 where the outward pointed and reversed flow areas alternate near the diffuser outlet. The number of these alternating regions exactly corresponds to the number of the prescribed jet-wakes at the diffuser inlet. In Figure 3 the prescribed jet-wake pattern can be clearly distinguished from the generated flow pattern near the diffuser outlet where the flow field consists of the alternating clockwise and counterclockwise rotating areas.

Relative velocity field of the two-dimensional rotating instability is shown in Figure 4 where the area in and around one rotating cell is shown. Rotating cells that already propagate around the circumference also rotate around their own axis. This velocity field clearly shows the counterclockwise rotation of the flow field within the cell and clockwise rotation of the flow field in between the rotating cells. The counterclockwise rotation of the cells is a consequence of the clockwise-prescribed rotation of the jet-wake pattern at the diffuser inlet. Also in this figure, the vortex structures coupled with the prescribed inlet flow conditions can be distinguished from the generated flow field near the diffuser outlet. 
TABLe 1: The stability limit.

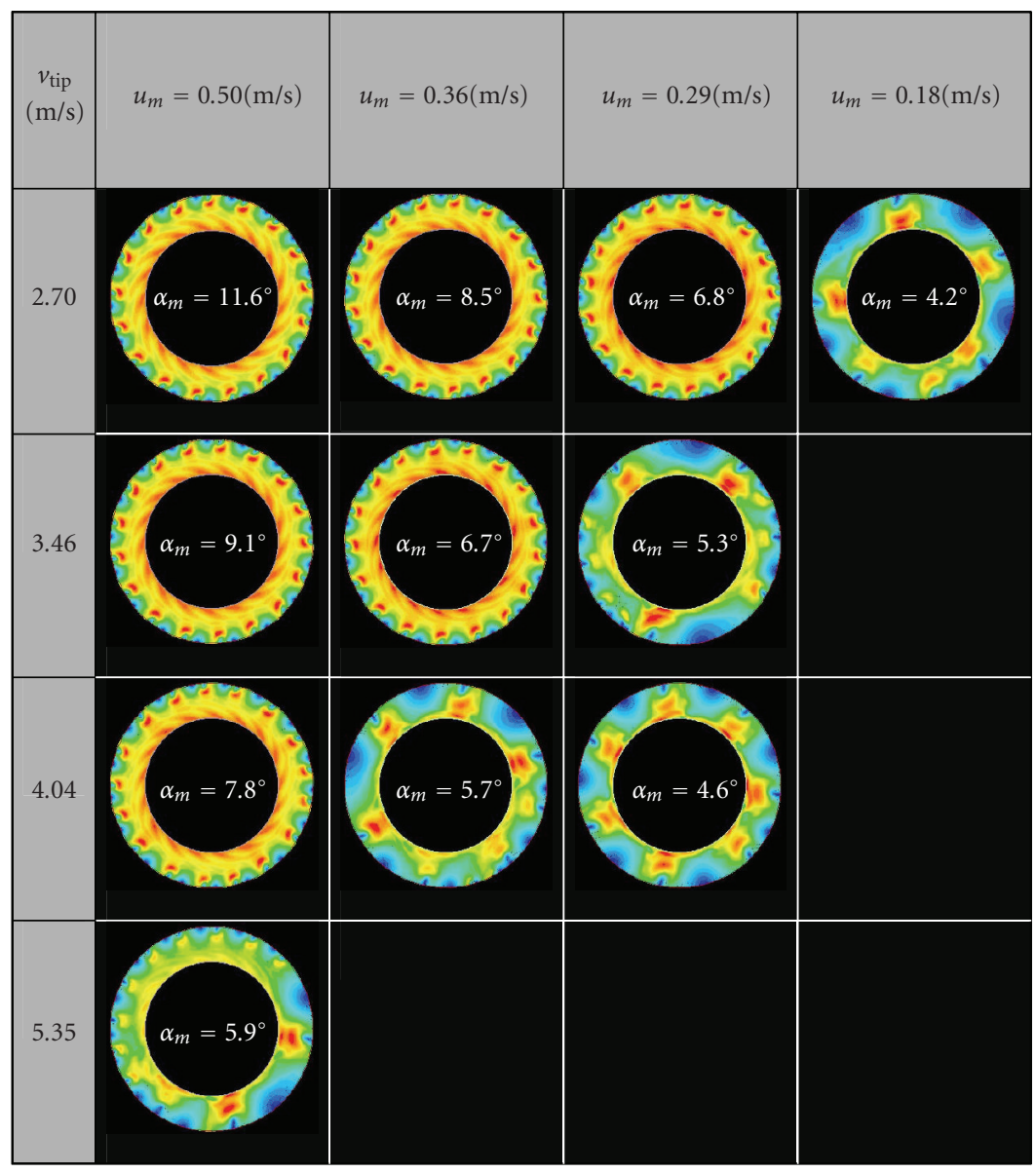

\section{PARAMETER ANALYSIS}

To study the influence of the diffuser geometry and the diffuser inlet flow conditions on the two-dimensional rotating instability, a parameter analysis is performed. Each time only one parameter is varied with respect to the reference diffuser geometry and flow conditions. The main varied features are the diffuser radius ratio, the number of impeller blades, and the shape and intensity of the jet-wake pattern at the diffuser inlet. The effect of different parameters on the critical flow angle, the number of rotating cells, their radial and circumferential extent, and their propagation speed is investigated. Whenever one parameter is varied, critical flow angle at the diffuser inlet $\alpha_{\mathrm{cr}}$, number of rotating cells $m$, and their relative propagation speed $\omega_{s} / \omega_{i}$ are monitored and analyzed. The estimated uncertainty in the critical flow angle and the propagation speed ratio is $\pm 0.1\left(^{\circ}\right)$ and \pm 0.02 , respectively. The explanation of the used symbols and indices for different parameters is given in Table 2.

\subsection{Diffuser radius ratio $\left(r_{3} / r_{2}\right)$}

To investigate the influence of the diffuser radius ratio on the vaneless diffuser core flow stability, the diffuser outlet radius is varied while the inlet radius remains unchanged. The influence of the diffuser radius ratio on the critical flow angle, number of rotating cells, and their propagation speed is given in Figure 5.

The critical flow angle is found to be strongly dependent on the diffuser radius ratio. As the diffuser radius ratio decreases, critical flow angle also decreases and the core flow stability of the vaneless diffuser improves. For large diffuser radius ratios, $r_{3} / r_{2}>2.5$, diffuser flow is found to be unstable or stalled for all flow angles.

The number of rotating cells and their propagation speed both decrease as the radius ratio increases. Corresponding solutions to the three investigated diffuser radius ratios are shown in Figure 6. Solutions in Figure 6 are represented by the velocity field colored by the velocity magnitude and they show the structure of the two-dimensional rotating instability for three different diffuser radius ratios. Figure 6 reveals that not only the number of rotating cells but also their radial and circumferential extent change as the radius ratio is varied. The number of rotating cells, their extent, and their distance from the impeller might have influence on the propagation speed of the rotating instability.

The number of rotating cells decreases as the radius ratio increases while the radial as well as circumferential extent of the cells increase. Larger diffuser space allows the cells to be larger in radial extent and it seems that the circumferential 


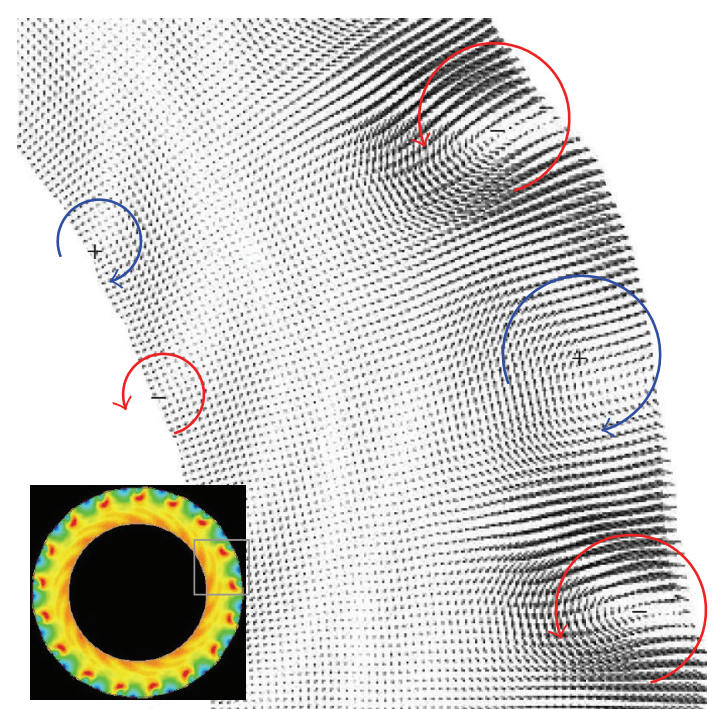

FIgURE 3: Relative velocity flow field corresponding to the stable operating flow condition.

and radial extent of the cells must be proportional to each other to obtain an on itself stable pattern of rotating cells. This extent proportionality is probably, together with the mass-flow rate, determinative for the occurring number of rotating cells.

Because only the diffuser outlet radius is varied while the diffuser inlet radius remained unchanged, the influence of the diffuser radius ratio indicates that not only the diffuser inlet flow conditions but also the diffuser outlet flow conditions have significant influence on the two-dimensional core flow instability.

\subsection{Number of impeller blades $(N)$}

The number of impeller blades is also varied. The influence of the impeller blade number on the two-dimensional rotating instability is shown in Figure 7 where the critical flow angle, the number of rotating cells, and their relative propagation speed are plotted versus the diffuser radius ratio. For $N=23$ and 25 the diffuser flow is found to be unstable for all mean flow angles at the diffuser inlet. Figure 7 shows that the number of impeller blades strongly influences the stability limit of the vaneless diffuser but no particular trend of the critical flow angle versus the number of impeller blades can be noticed. The number of the impeller blades also has significant influence on the number of rotating cells. Similar trend as that of the critical flow angle is obtained, which might indicate the existence of some link between the critical flow angle and the number of rotating cells. According to Figure 7 propagation speed of the two-dimensional rotating cells remains unchanged although the number of rotating cells strongly changes with the varying number of impeller blades. By varying the number of impeller blades, it is shown that the jet-wake pattern density around the circumference plays a significant role in the core flow instability within the

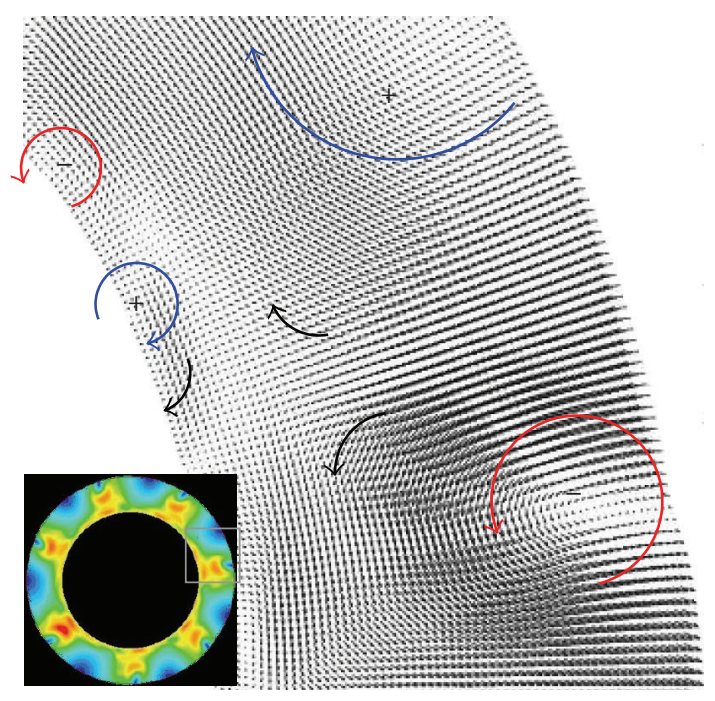

FIGURE 4: Relative velocity flow field corresponding to a twodimensional rotating instability.

vaneless diffusers but that it does not influence the propagation speed of the cells.

\subsection{Jet-to-wake intensity ratio $(a / b)$}

First varied parameter concerning the inlet flow condition is the so-called $a / b$ ratio, which is the ratio of the jet-to-wake intensity as defined in Figure 8 . The $a / b$ ratio is varied from 1 to 11.5 where $a / b=1$ is the no jet-wake situation.

The critical flow angle obtained for different $a / b$ ratios is plotted in Figure 9. The $a / b$ ratio has no influence on the critical flow angle until the low $a / b$ ratios are reached. At low $a / b$ ratios the critical flow angle slightly increases. Even when $a / b=1$, the two-dimensional rotating instability is observed. This means that the jet-wake pattern at the diffuser inlet is not essential for the occurrence of the two-dimensional rotating instability. According to Figure 9, the jet-wake seems to have a stabilizing effect on the two-dimensional core flow.

Together with the critical flow angle, the number of rotating cells also increases with the decreasing $a / b$ ratio as shown in Figure 9, but their propagation speed which is approximately $40 \%$ of the impeller speed remains unchanged. The number of cells increases from 7 to 8 as the jet-wake vanishes. According to Figure 7 the number of prescribed jetwakes around the circumference, representing the number of impeller blades, strongly influences the number of rotating cells. This means that the jet-wake is one of determining parameters for the number of rotating cells. But when jet-wake disappears, possibly other determining parameters, such as the characteristic length, for example, take over and the number of rotating cells changes.

Therefore, probably two different areas of influence can be distinguished. One when the jet-wake is strongly present, $a / b>3$, where $a / b$ ratio has no influence on the twodimensional rotating instability, and the other when the 
TABle 2

\begin{tabular}{|c|c|}
\hline Notation & Definition \\
\hline$A$ & Amplitude \\
\hline$a$ & Jet intensity \\
\hline$b$ & Wake intensity \\
\hline$c$ & Circumferential extent of the wake \\
\hline$c_{p}$ & Specific heat at $p=$ constant \\
\hline$d$ & Circumferential extent of the jet \\
\hline$D$ & Jet-wake transition steepness \\
\hline$h$ & Diffuser width \\
\hline$L$ & Diffuser length \\
\hline$m$ & Number of rotating cells \\
\hline $\mathrm{Ma}$ & Mach number \\
\hline$N$ & Number of impeller blades \\
\hline$p$ & Static pressure \\
\hline$p_{0 i}$ & Stagnation pressure, $i=1,2,3$ \\
\hline$r$ & Radius \\
\hline $\operatorname{Re}$ & Reynolds number \\
\hline$t$ & Time \\
\hline$T_{0 i}$ & Stagnation temperature, $i=1,2,3$ \\
\hline$u$ & Radial velocity component \\
\hline$V$ & Absolute velocity \\
\hline$v$ & Tangential velocity component \\
\hline$Y$ & Jet-wake shape and speed \\
\hline$v_{\text {tip }}$ & Impeller tip speed \\
\hline Greek letters & Defination \\
\hline$\alpha$ & Flow angle \\
\hline$\gamma$ & Ratio of specific heat \\
\hline$\eta_{c}$ & Overall isentropic efficiency \\
\hline$v$ & Kinematic viscosity \\
\hline$\theta$ & Circumferential position \\
\hline$\rho$ & Density \\
\hline$\sigma$ & Slip factor \\
\hline$\omega$ & Angular velocity \\
\hline$\psi$ & Power input factor \\
\hline Subscripts & Defination \\
\hline $\mathrm{cr}$ & Critical \\
\hline$i$ & Impeller \\
\hline$m$ & Mean \\
\hline$s$ & Stall \\
\hline tip & Impeller tip \\
\hline 1 & Impeller inlet \\
\hline 2 & Diffuser inlet \\
\hline 3 & Diffuser outlet \\
\hline
\end{tabular}

jet-wake is weak, $a / b<3$, where the two-dimensional rotating instability is probably besides the jet-wake also influenced by some other still to be determined parameters.

\subsection{Other parameters}

Besides the above-mentioned parameters, also the so-called $c / d$ ratio, the jet-wake function steepness $D$, and the additional velocity fluctuations as defined in Figure 10 are varied.

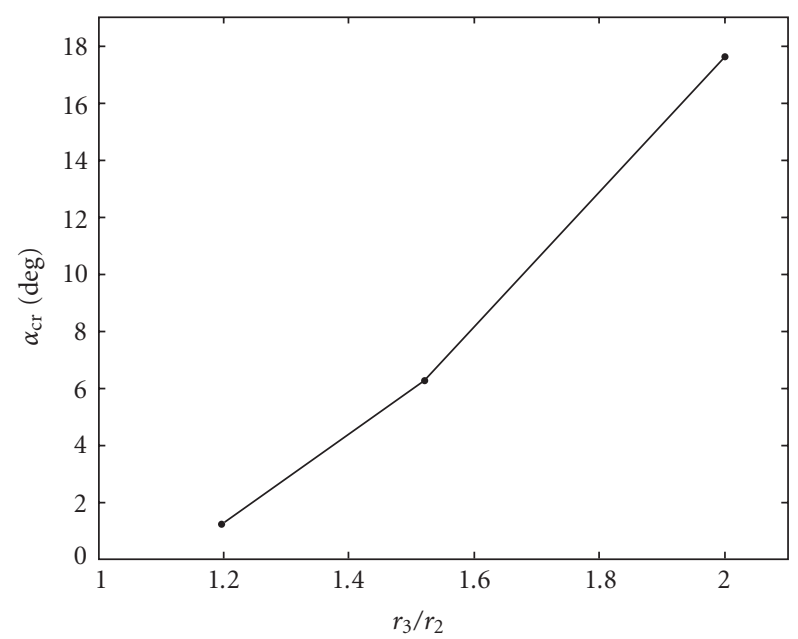

(a)

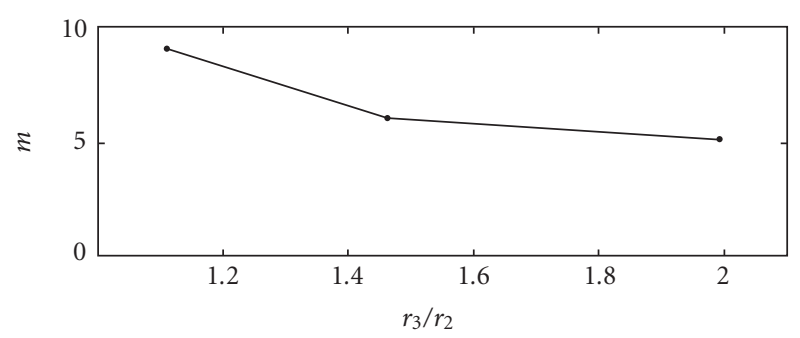

(b)

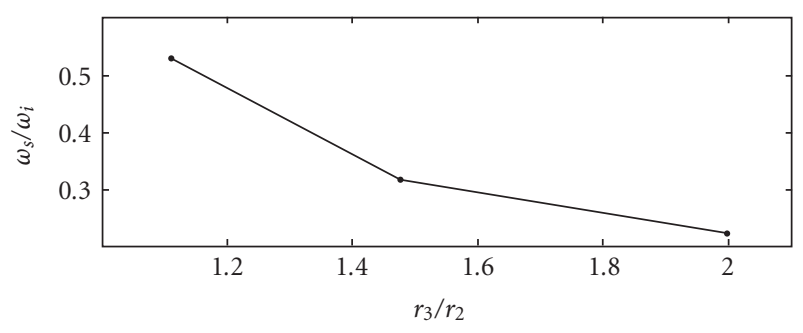

(c)

FIGURE 5: (a) The critical flow angle, (b) number of rotating cells, and (c) their relative propagation speed versus the diffuser radius ratio.

The $c / d$ ratio is the wake-to-jet ratio of their circumferential extent. Influence of these parameters on the core flow stability turned out to be negligibly small and therefore it is not discussed in this paper.

\section{DISCUSSION AND CONCLUSIONS}

A two-dimensional vaneless diffuser flow analysis is performed to study the core flow instability within the wide vaneless radial diffuser of centrifugal compressor. A twodimensional viscous incompressible flow model is developed within FLUENT where the influence of the wall boundary layers is neglected. Using this model two-dimensional rotating instability is obtained. To study the behavior and characteristics of this instability, different geometry parameters, 


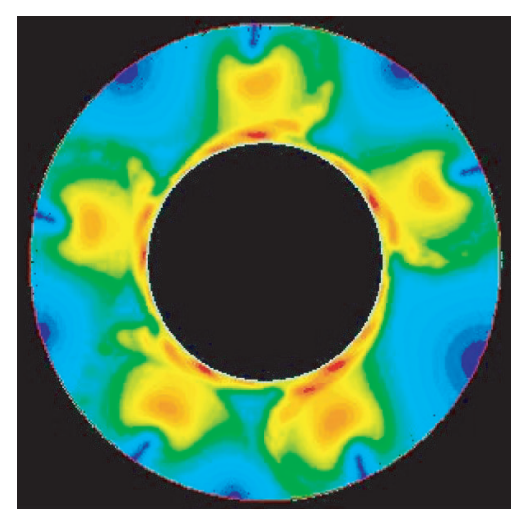

(a)

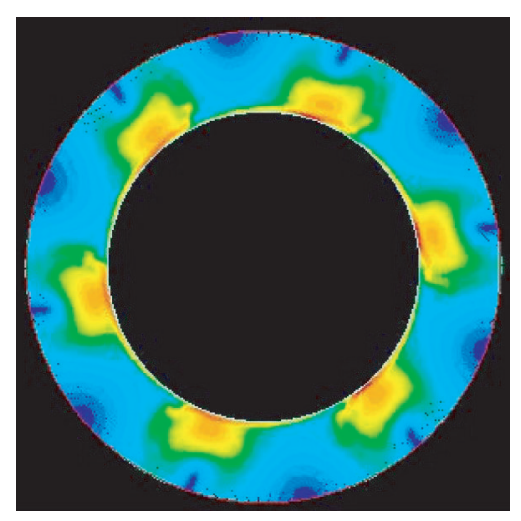

(b)

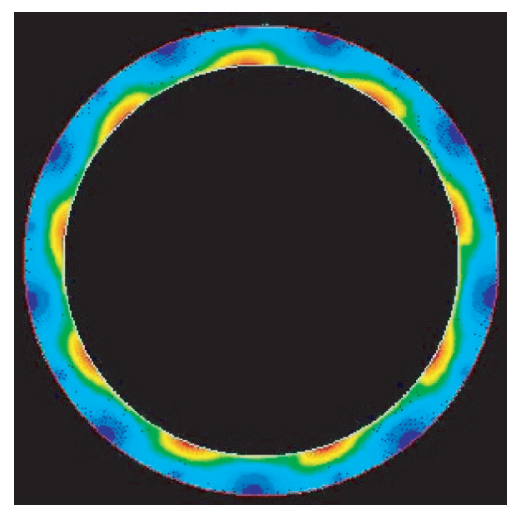

(c)

FIgURE 6: Solutions of the rotating instability for (a) $r_{3} / r_{2}=2$, (b) $r_{3} / r_{2}=1.52$, and (c) $r_{3} / r_{2}=1.2$.

inlet and outlet flow conditions are varied. Performed parameter analysis shows that the stability limit as well as the two-dimensional rotating instability are influenced by the configuration geometry and inlet and outlet boundary conditions. The results of this analysis are applicable only to the wide vaneless diffusers where the core flow dominates and the influence of the wall boundary layers can be neglected.

The two-dimensional rotating instability associated with rotating stall in wide vaneless diffusers is found to exist.

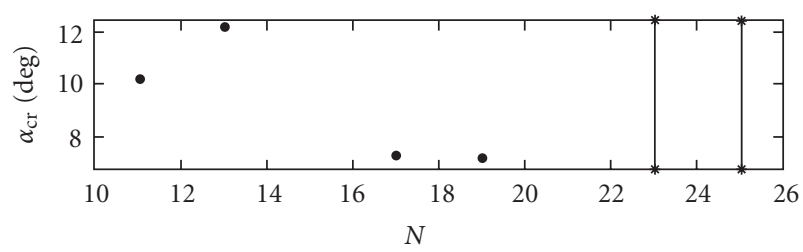

(a)

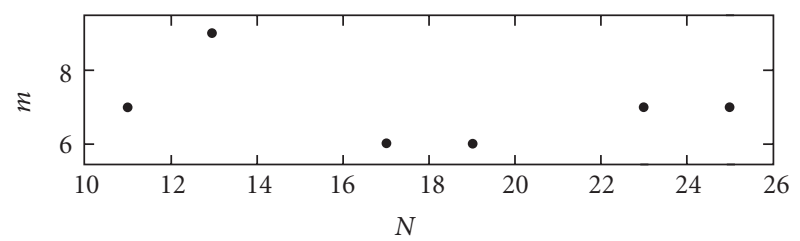

(b)

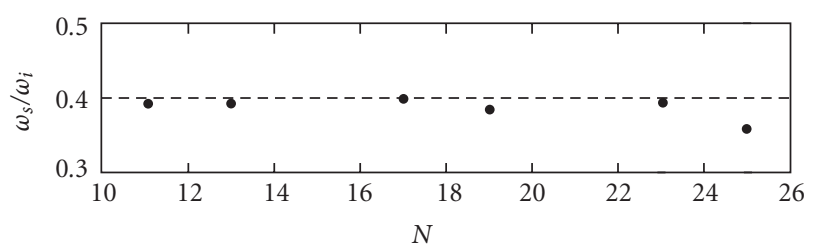

(c)

Figure 7: (a) The critical flow angle, (b) number of rotating cells, and (c) their relative propagation speed versus the number of impeller blades $N$.

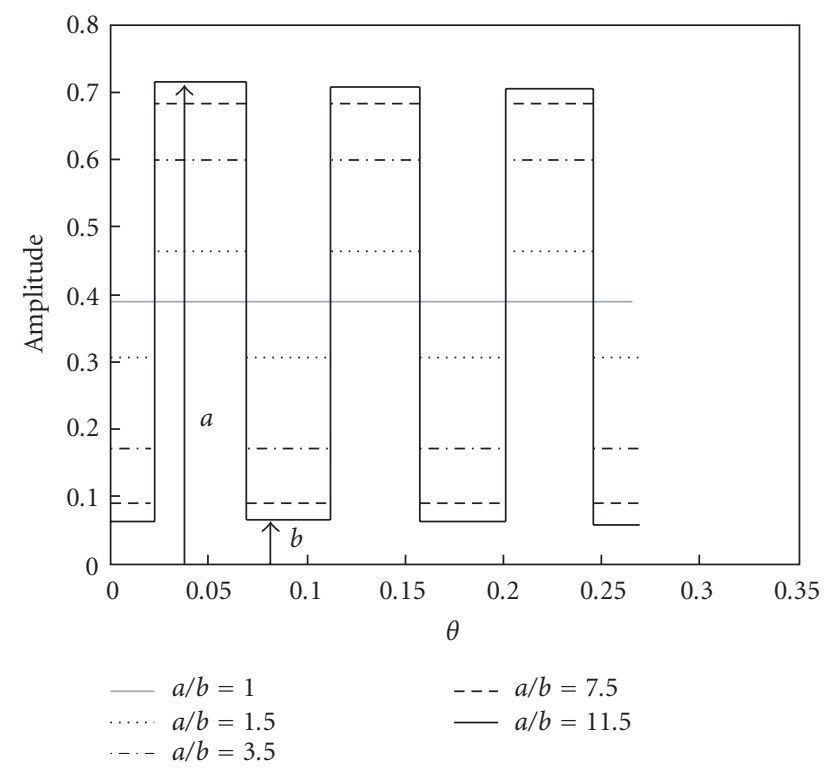

FIgURE 8: Varied $a / b$ (ratio of the jet-to-wake intensity).

The stability limit for wide vaneless radial diffusers, where the effect of wall boundary layers is negligible, can be expressed in terms of the critical flow angle. This two-dimensional rotating instability turned out to occur when the mean flow angle becomes smaller than the critical flow angle. 


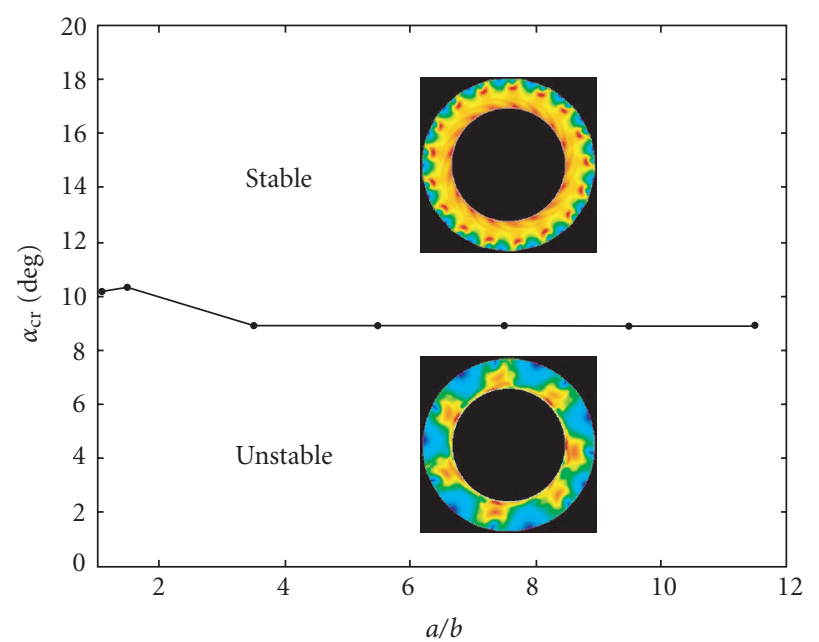

(a)

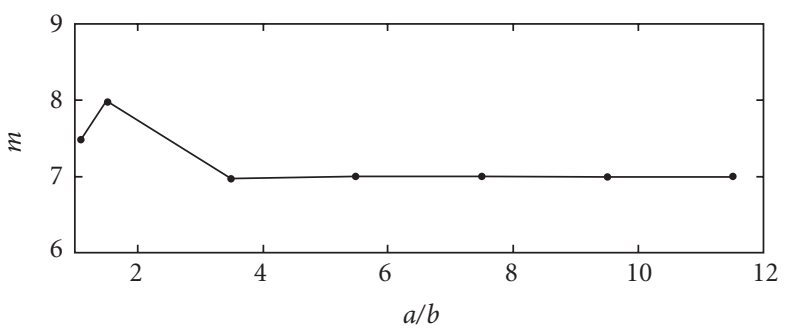

(b)

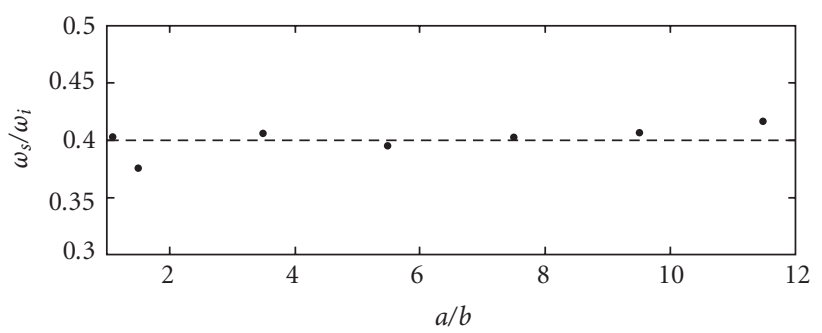

(c)

Figure 9: (a) The critical flow angle, (b) number of rotating cells, and (c) their relative propagation speed versus $a / b$ ratio.

The obtained two-dimensional rotating instability is similar to rotating stall in wide vaneless radial diffusers. It develops within four to eight impeller revolutions and consists of a number of rotating cells that are propagating with a fraction of the impeller speed around the circumference. This similarity and good agreement with data from the literature, as shown in [16], implies that this instability might contribute to the vaneless diffuser rotating stall.

The flow field analysis shows that the cells propagating around the circumference also rotate around their own axis in the opposite rotation direction than the impeller.

Numerical analysis shows that the diffuser radius ratio has significant influence on the critical flow angle, number of rotating cells, and their propagation speed. As the diffuser radius ratio decreases, critical flow angle also decreases and the core flow stability of the wide vaneless diffuser improves. The
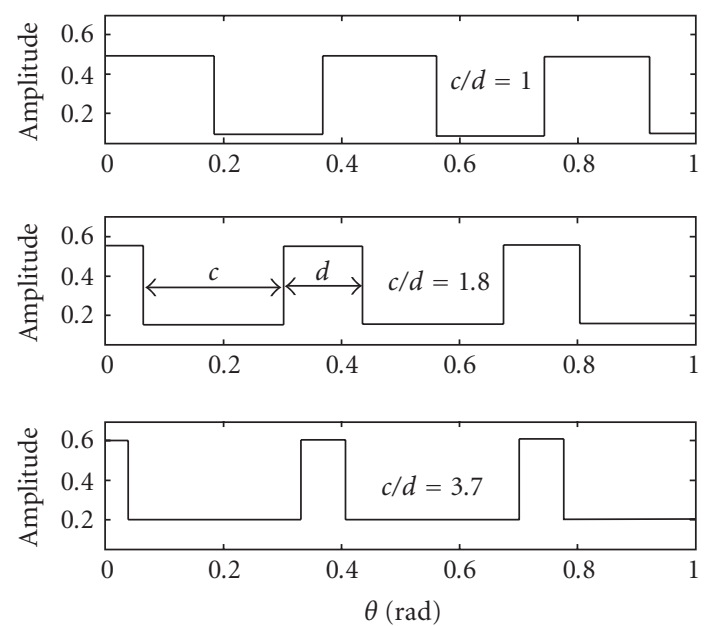

(a)

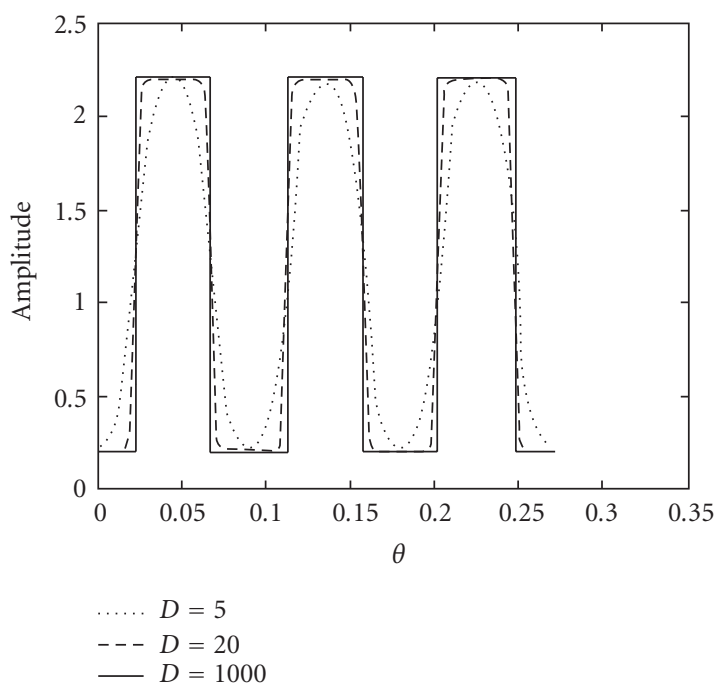

(b)
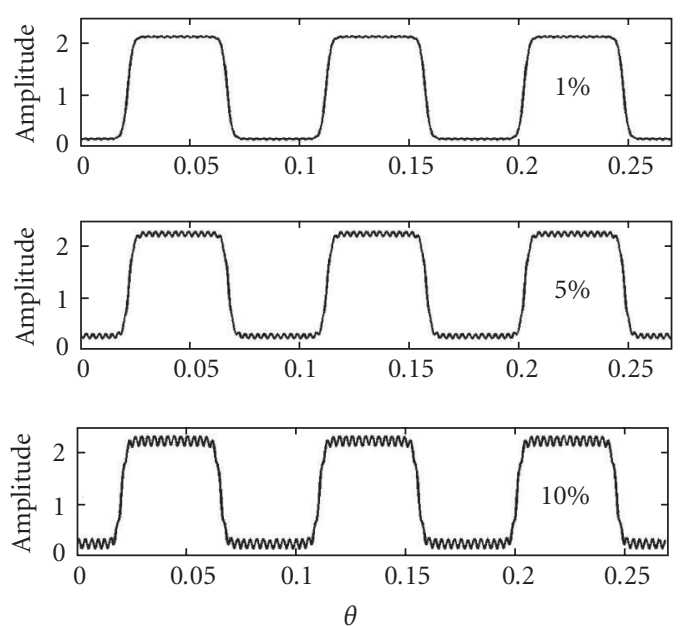

(c)

Figure 10: (a) $c / d$ ratio, (b) jet-wake function steepness $D$, and (c) fluctuation intensity. 
number of rotating cells as well as the propagations speed both decrease with increasing diffuser radius ratio. The influence of the diffuser radius ratio observed in the model is compared with the data from the literature in [16] and generally, a good agreement is found.

Strong influence of the diffuser radius ratio on the stability limit and the instability itself suggests that not only the inlet but also the outlet flow conditions have significant influence on the core flow stability. Since the outlet boundary condition has strong influence on the generated velocity field, it is a subject of further investigation.

The parameter analysis reveals that the number of prescribed jet-wakes around the circumference, representing the number of impeller blades, strongly influences the critical flow angle and the number of rotating cells. This implies that the two-dimensional rotating instability is dependent on the type of the impeller-diffuser configuration, which is in good agreement with the observations found in the literature. No particular trend is found between the critical flow angle and the number of impeller blades as well as between the number of rotating cells and the number of impeller blades. The propagation speed of the rotating cells remains unchanged as the number of impeller blades is varied.

The influence of the jet-wake intensity ratio $a / b$ on the two-dimensional rotating instability has two sides. On one hand, when the $a / b$ ratio is high, it has no influence on the two-dimensional rotating instability. On the other hand, the presence or absence of the jet-wake does make a difference. It seems that strong presence of the jet-wake stabilizes the vaneless diffuser core flow and that when the jet-wake becomes weaker, smaller $a / b$ ratios, the core flow becomes easily disturbed. When $a / b=1$, the two-dimensional rotating instability is still observed which indicates that the jet-wake pattern at the diffuser inlet is not essential for the occurrence of the two-dimensional rotating instability.

Two-dimensional rotating instability is very slightly influenced by the $a / b$ ratio, $c / d$ ratio, steepness of the jet-wake function, and the additional velocity fluctuations. This indicates that the shape of the jet-wake has negligible influence on the critical flow angle, the number of rotating cells, and their propagation speed compared to the influence of the diffuser radius ratio or the number of impeller blades.

According to the parameter analysis, stability limit of the two-dimensional vaneless diffuser core flow is strongly influenced by the mean flow angle, diffuser radius ratio, and the number of impeller blades. And it is influenced by the $a / b$ ratio only when $a / b$ is small. Therefore, the core flow stability of the wide vaneless diffusers can be improved by increasing the mean flow angle, decreasing the diffuser radius ratio, increasing the jet-wake $a / b$ ratio above 3 , and making a considered selection of the number of impeller blades. Although the $c / d$ ratio, the steepness of the jet-wake peaks, and the additional velocity fluctuations have negligible influence on the two-dimensional core flow stability, they can better be chosen such to support the diffuser stability. The $c / d$ ratio should be as large as possible, velocity fluctuations should be as low as possible and the jet-wake peaks should be as steep and sharp as possible.
The number of rotating cells is strongly influenced by the diffuser radius ratio and the number of impeller blades. The other varied parameters have negligible influence on the number of rotating cells and should not be considered. As the radius ratio increases, the extent of rotating cells also increases which results in less number of rotating cells around the circumference. No particular trend could be noticed between the number of rotating cells and the number of impeller blades.

Propagation speed of rotating cells does not seem to be influenced by any other parameter but the diffuser radius ratio. This is the only parameter where the size of the $r-\theta$ plane changes when being varied. It seems that only the diffuser geometry influences the propagation speed of the rotating cells. This can be related to the available diffuser length $L$, defined as $L=r_{3}-r_{2}$. The change in the radius ratio changes the radial extent of rotating cells. Since the radial and circumferential extents of the cells tend to be proportional, total size as well as the number of rotating cells around the circumference will change. Besides that, the larger the radius ratio, the larger the distance of the cells from the impeller. These characteristics might be determinative for the final propagation speed of the rotating cells around the circumference, but it is still a subject to be studied.

By prescribing the fixed inlet conditions, the interaction with the impeller is not taken into account in the current model. It is not known if the upstream impeller affects the vaneless diffuser stall or not. Abdelhamid [9] shows that stall pattern depends on the coupling conditions between the impeller and diffuser and Tsujimoto et al. [10] claim that vaneless diffuser rotating stall is nearly unaffected by the upstream impeller.

Since the vaneless diffuser flow is three-dimensional, the applied two-dimensional approach has its limitations. One of the uncertainties of two-dimensional modeling is that any three-dimensional flow mechanism that might be responsible for the occurrence of rotating stall cannot be detected with this model. Therefore, the model must be extended with the wall boundary layers at the hub and the shroud sidewall. It is expected that the addition of the wall boundary layer effects into the model will probably change if not the magnitude of the critical flow angle, then certainly the structure of the two-dimensional rotating instability, that is, the number and propagation speed of rotating cells. It is not known if the three-dimensional effects are more determinative for the stability limit or for the structure of rotating instability, but it is another subject to be investigated.

This analysis does not explain the flow mechanism of rotating stall, but it suggests a few of the physical phenomena that might contribute to the rotating stall mechanism in the wide vaneless diffuser. Current model points towards the possibility that the core flow instability might be one of the flow mechanisms responsible for the rotating stall in wide vaneless diffusers. Tsujimoto et al. [10], who investigated vaneless diffuser rotating stall based on a two-dimensional inviscid flow analysis, have also found that the flows in the vaneless diffuser have a two-dimensional, inviscid, and rotational flow instability. Besides these models, the experimental 
results of Tsurusaki and Kinoshita [19] also suggested that the instability of the main flow contributes to the onset of rotating stall in vaneless diffuser, which is in agreement with the two models.

To investigate the additional flow mechanisms within the wide vaneless radial diffusers, this numerical model will be extended to the model that includes the wall boundary layer effects. Besides that, a water model of the centrifugal compressor stage is made in order to reconstruct this two-dimensional rotating instability and perform measurements comparable with the numerical analysis.

\section{ACKNOWLEDGMENT}

TNO from Delft and Siemens Demag Delaval Turbomachinery B.V. from Hengelo are thanked for their contribution to this project.

\section{REFERENCES}

[1] F. P. T. Willems, Modeling and bounded feedback stabilization of centrifugal compressor surge, Ph.D. thesis, Technische Universiteit Eindhoven, Eindhoven, The Netherlands, 2000, printed by University Press Facilities.

[2] A. N. Abdelhamid and J. Bertrand, "Distinctions between two types of self-excited gas oscillations in vaneless radial diffusers," Canadian Aeronautics and Space Journal, vol. 26, pp. 105-117, 1980, ASME Paper 79-GT-58.

[3] H.-S. Dou, Investigation of the Prediction of Losses in Radial Vaneless Diffusers, 1991, ASME Paper 91-GT-323.

[4] Y. H. Shin, K. H. Kim, and B. J. Son, "An experimental study on the development of a reverse flow zone in a vaneless diffuser," JSME International Journal Series B, vol. 41, no. 3, pp. 546-555, 1998.

[5] W. Jansen, "Rotating stall in a radial vaneless diffuser," ASME Journal of Basic Engineering, vol. 86, pp. 750-758, 1964.

[6] Y. Senoo and Y. Kinoshita, "Influence of inlet flow conditions and geometries of centrifugal vaneless diffusers on critical flow angle for reverse flow," ASME Journal of Fluids Engineering, vol. 99, pp. 98-103, 1977.

[7] P. Frigne and R. Van den Braembussche, "Distinction between different types of impeller and diffuser rotating stall in a centrifugal compressor with vaneless diffuser," ASME Journal of Engineering for Gas Turbines and Power, vol. 106, no. 2, pp. 468-474, 1984.

[8] H.-S. Dou and S. Mizuki, "Analysis of the flow in vaneless diffusers with large width-to-radius ratios," ASME Journal of Turbomachinery, vol. 120, pp. 193-201, 1998.

[9] A. N. Abdelhamid, Analysis of Rotating Stall in Vaneless Diffusers of Centrifugal Compressors, 1980, ASME Paper 80-GT184; New Orleans, La, USA.

[10] Y. Tsujimoto, Y. Yoshida, and Y. Mori, "Study of vaneless diffuser rotating stall based on two-dimensional inviscid flow analysis," ASME Journal of Fluids Engineering, vol. 118, pp. 123-127, 1996.

[11] A. N. Abdelhamid, "Effects of vaneless diffuser geometry on flow instability in centrifugal compression systems," Canadian Aeronautics and Space Journal, vol. 29, pp. 259-266, 1983.

[12] G. Ferrara, L. Ferrari, C. P. Mengoni, M. De Lucia, and L. Baldassarre, "Experimental investigation and characterization of the rotating stall in a high pressure centrifugal compressor. Part I: Influence of diffuser geometry on stall inception,” in
Proceedings of ASME Turbo Expo, Amsterdam, The Netherlands, June 2002.

[13] G. Ferrara, L. Ferrari, C. P. Mengoni, M. De Lucia, and L. Baldassarre, "Experimental investigation and characterization of the rotating stall in a high pressure centrifugal compressor. Part II: Influence of diffuser geometry on stage performance," in Proceedings of ASME Turbo Expo, Amsterdam, The Netherlands, June 2002.

[14] A. Cellai, G. Ferrara, L. Ferrari, C. P. Mengoni, and L. Baldassarre, "Experimental investigation and characterization of the rotating stall in a high pressure centrifugal compressor. Part III: Influence of diffuser geometry on stall inception and performance (II impeller)," in Proceedings of ASME Turbo Expo, Atlanta, Ga, USA, June 2003.

[15] A. Cellai, G. Ferrara, L. Ferrari, C. P. Mengoni, and L. Baldassarre, "Experimental investigation and characterization of the rotating stall in a high pressure centrifugal compressor. Part IV: Impeller influence on diffuser stability," in Proceedings of ASME Turbo Expo, Atlanta, Ga, USA, June 2003.

[16] S. Ljevar, H. C. de Lange, and A. A. van Steenhoven, "Rotating stall characteristics in a wide vaneless diffuser," in Proceedings of ASME Turbo Expo, Reno-Tahoe, Nev, USA, June 2005.

[17] H. Cohen, G. F. C. Rogers, and H. I. H. Saravanamuttoo, Gas Turbine Theory, Addison-Wesley Longman, Harlow, UK, 4th edition, 1996.

[18] B. P. Leonard, "A stable and accurate convective modelling procedure based on quadratic upstream interpolation," Computer Methods in Applied Mechanics and Engineering, vol. 19, no. 1, pp. 59-98, 1979.

[19] H. Tsurusaki and T. Kinoshita, "Flow control of rotating stall in a radial vaneless diffuser," ASME Journal of Basic Engineering, vol. 123, pp. 281-286, 2001. 

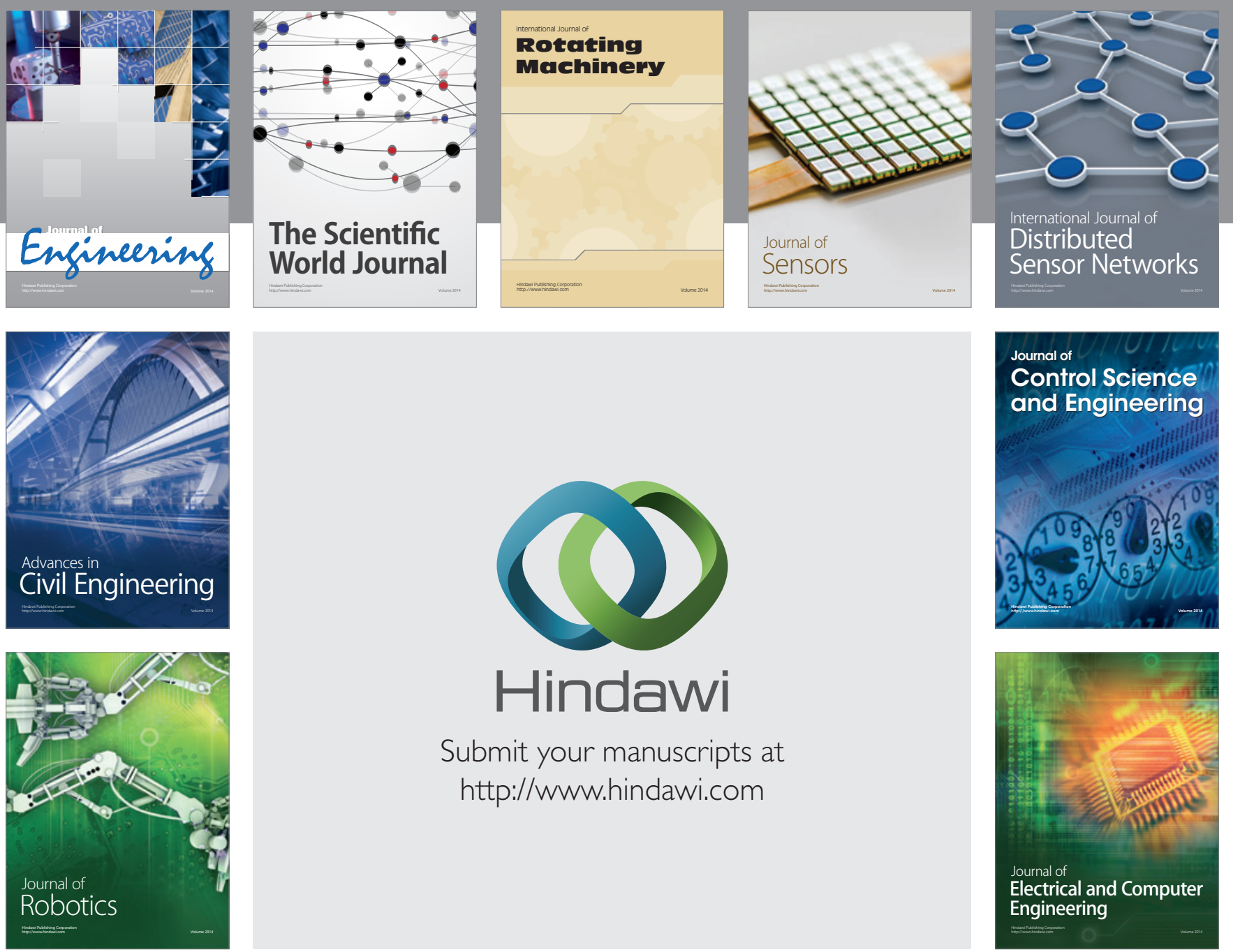

Submit your manuscripts at

http://www.hindawi.com
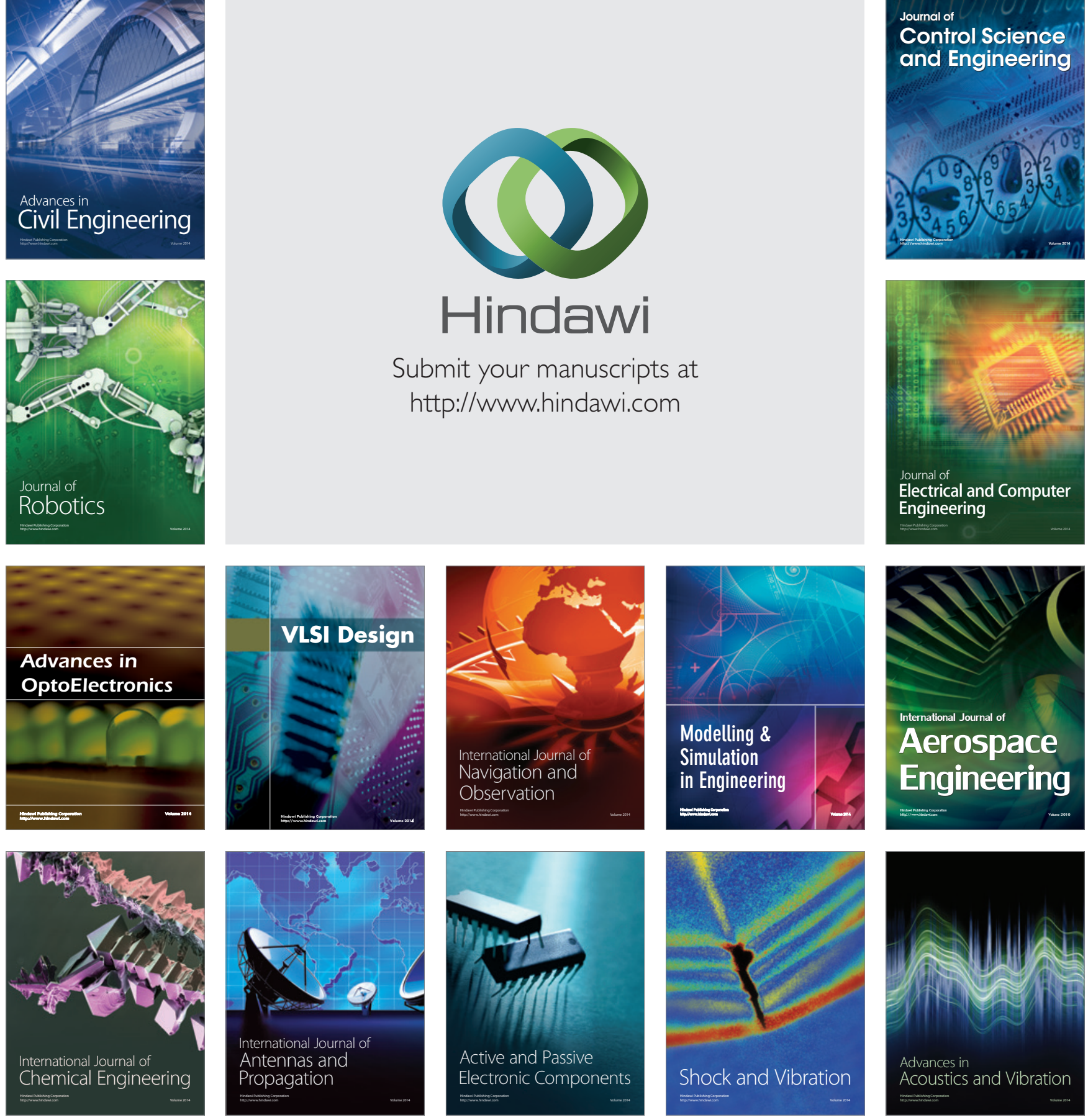